\title{
Light-sheet microscopes unwrap the heart
}

\author{
elife 6, e28307 (2017)
}

A heartbeat is a carefully orchestrated event. Pacemaker cells direct the rhythmic contraction of neighboring cardiomyocytes that pulse the heart in the correct sequence to pump blood throughout the body. The organogenesis leading to those coordinated calcium waves that produce each beat is itself a series of harmonized events. At some point, cells destined to become part of an intact, functioning organ start signaling to each other, as development proceeds from a cluster of cells to simple sheets to the fully formed, multichambered heart.

In the past, tracking cells in the developing heart has been limited to ex vivo snapshots. Patch clamps can detail the activation and deactivation of cardiac cells grown in culture, though only one cell at a time. Optical mapping with injected dyes can look at what's going on in more intact tissues, but necessitates removing the heart from the developing organism. The actual cell-to-cell sequences of conduction during organogenesis of the heart had remained a mystery. But a new technique is finally shining light on it.

Jan Huisken describes himself as a physicist with an interest in answering biological questions too. During his $\mathrm{PhD}$ with Ernst Stelzer at the European Molecular Biology Lab in Heidelberg, Germany, he began engineering light-sheet microscopes, with the goal of improving the imaging of large specimens. Rather than shining light from above, light sheets illuminate a single plane from the side; this reduces the risk of phototoxicity and allows a specimen to be imaged from different directions and for longer periods of time than conventional microscopes. Fellow biologists on hand in Heidelberg helped test the developing technology with organisms like Medaka and Drosophila, and liked the possibilities. "[We realized] that the system is not only quite nicely designed from the optics points of view but also extremely useful for the biologist," says Huisken. A post-doc took him to the University of California, where he adopted the zebrafish as a model organism and cultivated an interest in vertebrate development. "With a zebrafish we are kind of at a sweet spot," Huisken says. "We are looking at a vertebrate system; we have a two chambered heart; we have a transparent organism; we can do all the in vivo microscopy."

In his latest paper, Huisken and colleagues unwrap the larval zebrafish heart

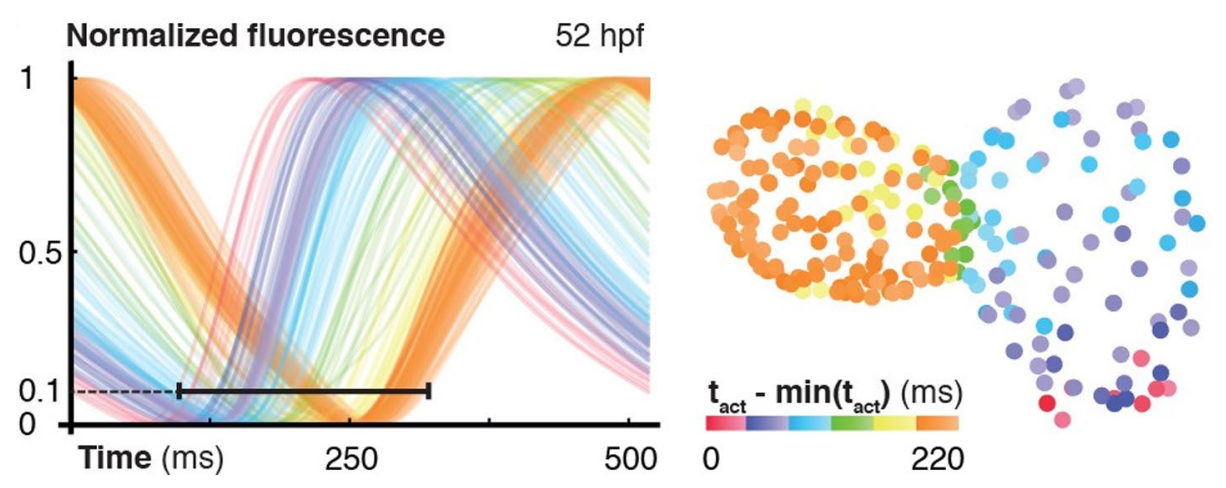

Normalized fluorescence of each calcium transit from individual cells. Credit: Image from eLife 2017; 6:e28307

and for the first time observe how individual cells are connected as they propagate calcium signals across developing tissues. The technique used was based on a recent Nature Methods paper, where Huisken demonstrated that light-sheet microscopy with the right data analysis could produce detailed, multimensional reconstructions of the larval zebrafish heart (Nat. Methods 11, 919-922; 2014). From those first movies, the lab became "more interested in the functional aspects and how the cardiac conduction travels," he says. They labeled each cell with a nuclear marker to determine its location and with a calcium indicator to follow the calcium transits of each beat. But as easy as the translucent larval zebrafish are to image, the physical movement of the beating heart still complicates the process. Fortunately, cardiologists already had a solution for that problem: a zebrafish line with a silent heart. "The heart is not beating but the cardiac conduction is perfectly intact," Huisken explains; zebrafish are actually small enough to survive the first few days of development without a beating heart.

But all hearts are not created equal; hearts from different fish will each have slightly different shapes, a problem when observing conduction, not morphology, is the goal. The light sheet technique also records immense amounts of multimensional data-terabytes, Huisken says-so they needed a way to visualize where cells were and what they were doing in a straightforward way, without the subtle variances in shape from fish-to-fish getting in the way. So instead of analyzing complicated 3D images, they established a novel coordinate system to arrange each individual cell onto a simple cylinder, thus eliminating any morphological differences.
That cylinder could then be unwrapped and analyzed in just two dimensions.

With the approach established, the team could then observe the conduction patterns across each cell during early development and after the heart matured into a twochambered organ. Albeit a very small one. "It's two hundred microns across, it's smaller than a human hair," says Huisken, with only a few hundred cells total. They saw that, rather than a sophisticated system to prevent retrograde flow before any valves developed, the cells that make up the heart when it's still a tube in early development are simply different shapes in different regions. As a result, the wave propagates quickly in some areas but then slows in others, establishing the careful timing of the heartbeat. As valves develop later, they just make the whole pattern more robust. "I think for me as a physicist the fascination there is primarily to see how the heart developed over time and how these cells managed to create something that one would think as probably optimal for the fish," says Huisken.

In his current lab at the Morgridge Institute for Research in Madison, Wisconsin, Huisken will continue his work on understanding organogenesis, with a bio-inspired eye towards engineering better pumps or even artificial organs. There's also work to be done to advance the capabilities of light-sheet microscopes and the computational considerations needed to analyze all those images they capture. Smarter microscopes still wait in the future.

Ellen P. Neff

Published online: 23 April 2018 https://doi.org/10.1038/s41684-018-0063-2 